\title{
Understanding Risk: Psychosis and Genomics Research in Singapore.
}

\author{
AYESHA AHMAD, TAMRA LYSAGHT, LIU JIANJUN, MYTHILY \\ SUBRAMANIAM, TAN SAY BENG, BENJAMIN CAPPS ${ }^{1}$
}

\begin{abstract}
This is an exploratory paper of the ethical implications for genomic research and mental illness with specific reference to Singapore. Singapore has a unique context due to its social and political systems, and although it is a relatively small country, its population is religiously and culturally diverse. The issues that we identify here, therefore, will offer new perspectives and will also shed light on the existing literature on psychiatric genomics in society. We contextualise issues such as risk and stigma in the identification and diagnosis of psychosis in the way they relate to Singaporean society, and use a current study (LYRIKS) as a case example.
\end{abstract}

Genomic research has the potential to change significantly he practice of clinical medicine if, as expected, fast and inexpensive sequencing becomes a reality. It will likely also change how society thinks and acts in respect to multi-factorial diseases, conditions, traits, and syndromes that have a genetic component. Genomic research already raises a number of ethical concerns relating to the privacy of individuals, including the disclosure of research results and incidental findings, surreptitious tests, third party access to data, and the re-emergence of genetic determinism. These issues are potentially exacerbated when genomics - the study of whole genomes to understand complex illness and behavioural traits - is applied to psychiatric research, because of the stigma that is often attached to mental illness. In this paper, we discuss some of the issues that have arisen in the context of a study in Singapore that is currently investigating the genomics and biomarkers of psychosis. We argue that although a genomic study rarely creates data that is directly useful to the participant, it can have incidental benefits to the individual who is identified during the study as being at high risk of developing psychosis and its related states. Understanding these potential benefits requires us to examine the implications that this type of research may have on public understandings of genomic data and risk.

\section{Introduction}

There is a growing research literature supporting a clinical model for earlyintervention treatment of those who, through a combination of modalities, might strongly be expected to develop clinical psychosis. ${ }^{2,3}$ This 'ultra high risk' (UHR) state is often indicated by a prodromal phase before psychotic breakthrough, and insights into behavioural changes and biomarkers have enabled timely treatment with more favorable clinical outcomes. This paper refers to ongoing genomic research that is likely to have a significant impact on mental health by increasing the understanding of complex illnesses and the potential implications of linking genomic screening within research populations for these prodromal indications. Genomic studies are now 
alluding to the heritable features of psychiatric illness and the expectation is that the genetic indicators may be allied with existing diagnosis tools to provide clinical preventative options. This possibility raises important ethical issues in the classification of the premonitory phases of psychosis.

However, by focusing on the psychopathology and patho-physiology of 'at-risk' states of psychosis, the research may also have implications for the timing at which the research participant seeks medical advice, his or her own recognition of symptoms, and his or her awareness of the significance of a history of familial diagnoses. These will also be affected by the public's understanding of risk and mental illness (here referring to, but without distinguishing between: the population of Singapore, its cultural enclaves, discrete communities, and the participant's family) because such relationships will shape and influence the context of the participant's experiences. In this paper, we examine mental health and 'clinical risk' in the context of a comprehensive investigation into the prodromal significance of psychosis that is currently underway in Singapore. As part of this study, researchers are focusing on identifying biomarkers associated with an 'at-risk' status. Rather than addressing the well-trodden issues of individual genetic identity, such as privacy or discrimination, it is our intention to consider the social implications of refining a clinical 'at-risk' genomic profile. Our analysis will provide an ethical point of view in respect to the conduct of genomic research in mental health.

\section{Background}

Psychiatric disorders have debilitating effects on the individual and their family, and a cost to wider society. However, the biological, environmental, and sub-threshold clinical features of mental conditions that precede full-blown illness are poorly understood. ${ }^{4}$ Psychoses, such as schizophrenia, begin with a prodromal period of altered functioning and symptomology before the onset of threshold illness. ${ }^{5}$ Currently, the prodromal phase is identifiable up to 30 months prior to the display of symptoms. ${ }^{6}$

Understanding causal patterns in psychiatric illness plays a crucial role in patient awareness of their condition and in clinical decision-making. ${ }^{7}$ There have been many approaches used in identifying an UHR state. These approaches, which focus on altered functioning and symptomology, have contributed to psychiatric tests which may identify individuals who are at risk of developing psychosis, albeit with a limited degree of confidence due to the potential for false positives, which in turn are related, to some extent, to the efficacy of recruitment of potential UHR individuals. ${ }^{8}$ These tests have most recently been combined with research into neuro-cognitive biomarkers and experimental brain imaging. Moreover, the latest high-throughput genomic analysis techniques, defined in terms of "the study of genes and their functions, and related techniques", 9 are now alluding to gene expression profiles that can be linked to specific patterns of clinical progression. ${ }^{10}$ 
Although the significance of genomics for psychiatric medicine is potentially substantial, the impact of attributing a genetic basis to certain psychiatric disorders is also a concern, especially if the association encourages prejudice towards patients in ways that diminishes their self-esteem and social or economic opportunities. The genetic provenance of illness may also increase apathy as a manifestation of 'determinism' or 'essentialism', which describes a reaction predicated on the notion that nothing can be done, or that one is fated to develop a particular illness because of genetic 'hardwiring'. ${ }^{11}$ Genomic research may also 'de-stigmatise' mental conditions by showing a 'shared heritage': that is, reaffirming the 'we' are 99.9\%-the-same' claim, ${ }^{12}$ or, in respect to a persuasive reclassification of psychiatric of disease so that communities can move away from the archaic 'moral' categories of deficiency. ${ }^{13}$

Severe psychotic disorders like schizophrenia are stigmatised across many different contexts. These disorders are often misconstrued as a socially determined 'weakness of character' and used to isolate people as inadequate, unlikable, and dangerous. ${ }^{14,15}$ As a result of finding clinical and empirical roots for mental illness, we might find that people may be more willing to come forward for treatment because it is easier for them to pinpoint a biological (and therefore more concrete) basis for their mental health problems, ${ }^{16,17}$ rather than attributing it to a 'social' weakness. An illness model of mental health is likely to lead to clinical intervention, rather than mere reflections upon the struggles (and failures) of social living.

However, in respect to genomic research, predicting health outcomes will largely be focused on population effects (for example, the expected reaction of a population to a particular drug) rather than an individual's options and opportunities (which may even be obscured). This creates a different ethical perspective from that which analyses the implications of genetic research. In many contexts, genetic tests allow individuals to make informed decisions about their clinical options, such as avoiding certain risky lifestyles or assisting in family planning. For example, a meta-analysis of the effectiveness of genetic testing for smoking-related diseases on smoking cessation found that the test resulted in higher risk perception and greater motivation to quit smoking, ${ }^{18}$ although some have questioned the proper use of such testing. ${ }^{19} \mathrm{~A}$ successful outcome is likely to be dependent in part on an individual's access to information (including genetics), as well as positive changes in health-related behaviour patterns.

Of note, however, is the predictive nature of genomic studies - that is, how population-wide studies will become 'personal' in terms of an individual's heritage, and family and community relationships. Thus, we can expect that should genomics eventually reveal UHR profiles, clinical tests will one day be used as decision aids in personalised diagnosis and treatment. However, what is presently unclear is how knowledge of genomic risks will affect the decisions typically made by, and on behalf of, prodromal individuals. This is because indications for 'at-risk' states are not a clinical entity. ${ }^{20}$ It is also unclear how families or communities may react to states which have an uncertain course and prognosis, or how the same information will be seized upon by 'interested' parties. The concern, therefore, is whether a deceptively 
simplistic model of psychosis, ${ }^{21}$ enabled by genomic studies, will reinvigorate deterministic ideas.

\section{Genomic Research in Singapore: The Longitudinal Youth at Risk Study (LYRIKS)}

LYRIKS is a five-year prospective observational study that began in 2009 and is being led by the Institute of Mental Health (IMH) in Singapore as part of a multicentre and multi-national study in translational and clinical research in psychosis.

The study aims to identify biomarkers of psychosis vulnerability and to develop a clinical model that provides strong positive identification of UHR individuals. The expectation is that individuals who are identified as UHR may be given prospective treatment during the clinically important prodromal stages and thereby potentially alter the course of their illness. ${ }^{22}$ While it must be noted that an ideal genetic marker for psychosis would be derived from patient populations rather than at-risk populations, this research raises important ethical considerations that need to be explored both for this particular context and for future projects.

For this study, researchers have been recruiting a cohort of young individuals aged between 14 to 29 years. Participants have been recruited from various sources including the IMH, schools, universities, the armed forces, counselling centers, and the community at large. Prospective participants are assessed for UHR using the Comprehensive Assessment of At Risk Mental States (CAARMS). Those who fulfill the criteria for UHR are then asked to participate in the study, and those who do not are invited to join the control group. Under informed consent, participants in the study group provide blood for genomic and lipidomic analyses, and are followed over two years with bi-annual clinical, neurocognitive and neuro-imaging assessments. They remain in the study either until they complete the two-year period of observation, choose to withdraw, or convert to psychosis.

\section{Addressing ethical issues in LYRIKS for the participant and clinician/ researcher relationship}

After the assessment for 'at risk status', participants found to meet criteria are informed that they are at a higher risk of developing a mental illness than the general population. They are also informed of any incidental findings, which are assessed to be of potential clinical significance by a neurologist who is part of the research team for those participants undergoing Magnetic Resonance Imaging. Since to date no biomarkers have been identified for psychosis, however, the results of the genomewide association studies/gene expression/lipidomics and other imaging parameters are not conveyed to the participants.

It is important to understand that while the IMH has a programme for young people who are at risk of developing mental illnesses - the Support for Wellness Achievement Program (SWAP) - the participants of LYRIKS are from a number of 
different sources, including other psychiatric out-patient services and self-referrals following our outreach campaigns. Thus, only some participants will be undergoing treatment. In treatment scenarios, consent is neither sought nor taken by the treating clinician, but is taken by a trained researcher aware of the potential for coercion and therapeutic misconception. For those participants who are not undergoing any treatment, LYRIKS provides a resource brochure that indicates contact numbers of various sources of professional help.

In this paper, we focus on the part of the study that will identify genomic polymorphisms associated with the development of psychosis and determine their predictive value (along with other biomarkers) in the prevention of conversion. The LYRIKS raises a number of ethical issues pertaining to informed consent and notions of the 'mature minor', the duty to provide clinical interventions in research, and the separation of research duties, which are discussed elsewhere. ${ }^{23}$ This part of the study will create large amounts of data - including genomic profiles - pertaining to the risk that young adults who meet certain conditions face. Notwithstanding the complexities that researchers will face in relating such profiles to the prodromal signs of mental health, here we address two particular challenges raised by the generation of such data in terms of determinism and a specific research cohort.

First, the prodromal signs of individuals who are identified as UHR will be correlated with genomic profiles by the research team. Without any interest in identifying these individuals, this part of the research is aimed at elucidating the genomic evidence for clinical judgments about the severity and likelihood of disease prognosis and prevalence. However, as mentioned already, misunderstanding genetic data risks misleading perceptions of psychiatric illness. Hence, it is foreseeable that the probability of conversion to psychosis will be interpreted and acted upon differently according to how the uncertainties of the research instruments are explained and subsequently understood. These variances may impact on the success of the predictive model.

Identifying clinical, neurocognitive and imaging markers, may also, in some cases, pose difficulties in forming a predictive model. For example, should UHR individuals be identified at an early enough stage to seek preventative treatment, their condition may not develop into psychosis and will thus appear to contradict the findings of the predictive model. Consequently, the research team will have to convey to the participants and their caregivers the idea of statistical relevance - the kind of information that has been the driver for deterministic ideologies in the past - given that all invited individuals, on the basis of meeting some of the criteria that might increase their risk of developing a mental illness, will prospectively be identified as 'ultra high risk'.

Second, the genomic study will potentially reveal data about certain demographics in the Singapore population with respect to a complex genetic disease. The implication for the LYRIKS is that it will eventually make possible the identification of individuals within the population who have ascertainable risk factors that can be 
linked to the prodromal phase. Such prospective identification may create further circumstances of stigma and exclusion on an individual basis. Genomic studies may shift the potential assumptions about determinism to populations, especially if genetic 'confirmation' of an illness is likely to lead to social exclusion.

\section{Understanding 'Risk', Clinical Data, and Genomics}

Diagnosing mental health conditions has potential psychosocial risks for those who are told they are 'at risk'; ${ }^{24}$ clinically, this might include a 'potential', 'uncertain', and 'possible' diagnosis. In terms of social harms, we only need look to the implications of genetic determinism. ${ }^{25}$ Despite an outmoded social and political context that supported the worst eugenic atrocities, the contemporary 'ideologically clean' and statistically verifiable idea of genetic determinism has gained some support. ${ }^{26}$ Determinism now suggests that some dispositions to behave in certain ways are indeed beyond our control and cannot wilfully be changed (including perhaps those behaviours resulting from mental health conditions).

If a UHR status results in only certain behaviours, it appears that determinism carries a negative weight: we are normatively classifying people's actions and behaviours in terms of what they are (and are not) responsible for. In terms of health, the 'dice have already been rolled'. However, this use of interpretative evidence has, for some, resulted in a "fruitless controversy" that can mislead social perceptions about, and shape unnecessary interventions in genetic health, because of a fundamental disparity about how we are to perceive ourselves as agents: both as autonomous (as genetic and ostensibly free beings) and moral (that we can make decisions about how to act) (see: Meaney $2001^{27}$ ). We can thus also find that an UHR status - although pertaining essentially to a risk status - can have positive effects in respect to personal opportunities, such as seeking help, watching for early signs, and preventative treatment.

Risk may potentially, therefore, affect an individual's sense of their own prognosis (or potential deterioration), in addition to shaping their role in their family and society. While some of the various issues we raise below could be applicable to genomic data from other diseases as well, creating genomic data in psychosis research has specific challenges related to the interest, care, and perception of individuals, their family and their caregivers, who are attempting to understand the nature of a UHR status generated by such research. Other parties will also want to know the status of individuals because of the implications for insurance or work, or out of simple curiosity. For the individual, there will likely be degrees of reaction, falling between apathy and a desire to find out the chances of developing the disorder, the implications of a positive diagnosis, the clinical interventions available to minimise developing illness, and approaches that may alleviate or treat the illness should progress. However, characterising or labelling people with an at-risk status can become stigmatising and can divest individuals of further opportunities. It is also possible that awareness of the hereditary factors may fuel prejudice, resentment, or hostility within or towards a discrete group. 
Within the context of LYRIKS, which involves participants of multi-ethnic backgrounds vulnerable to existing discriminations and projections, the subject of stigma is difficult to address. This issue is to be explored in future qualitative studies at the IMH which place an emphasis on the participant's narratives. Narratives form a crucial part of understanding any impact or influence of stigma related to mental illness, although it is difficult to be definitive because the distress faced by young individuals seeking help in psychiatric clinics is confounded by the nature of their symptoms. Internalisation of stigma may be related to the attenuated psychotic symptoms or the associated anxiety and depression, and in most cases, stigma is thus not introduced by the study.

Furthermore, in addition to the anxiety felt by an individual about his or her own riskstatus, both within and outside a family network, there is a wider concern about how data - whether clinically relevant or not - is understood by the public and other parties beyond the family or community, such as medical insurance companies, commanding officers, employers, and peers. Privacy rights, for example, have been campaigned for in ethical discourses that explore arguments about whether genomic data should be regarded as an exceptional form of information and knowledge. ${ }^{28}$ Much has been made of the inevitability of genetic discrimination and social disadvantage. Yet a counter-argument emphasises the need to share genetic information across affected populations to increase opportunities for all, which in itself is an ethical necessity for achieving a high standard of public health care. The ethics surrounding genomic data in psychosis requires a robust level of management between various members of research and clinical teams. We will shortly say a little more about these groups - individuals, communities and researchers.

\section{Understanding the Role of Researchers}

We find that there are a number of important roles that the researchers must undertake in addition to conducting their investigations competently and ethically. First, in addition to creating genomic data, researchers will make interpretive use of it. This use should be accompanied by considerations (and apprehensions) about scientific interpretation and clinical translation, and assessment of appropriate ways to facilitate communication to the patient (and taking a step back to the current study, the patient is also a research participant, which has implications for the dual responsibilities of clinician-researchers), and potentially to family members, communities. Finally, researchers should consider public engagement because of the wider implications of certain findings to populations.

Secondly, researchers who conduct genomic studies should be aware of the circumstances that encourage the use of genomic science as a means to demarcate population differences. For example, the recent development of an 'AfricanAmerican' only drug seeks to provide equity to certain members of a particular society. ${ }^{29,30}$ In light of such developments, there is a growing interesting around the world in investigating the potential of personalised medicine to reduce the effects of 
mental illness. At the same time, the difficulty is that underlying social and cultural conceptions about mental illness can have "dramatic consequences for help seeking, stereotyping and the kinds of treatment structures we create for people with mental illness". 31 The efficiency of mental health treatment clearly has grounding in the level of research into mental illness. Our case example, LYRIKS, evidences this by designing culturally appropriate conditions for confidentiality, especially for minors (in Singapore under 21 years of age) when investigating individuals who may be UHR. There is an important connection between the underlying social processes of the experience of a psychiatric disorder - both for the patient and their caregivers and the subsequent seeking of testing, diagnosis, and ultimately, clinical treatment.

Cultural, philosophical, and religious values contribute to more severe forms of stigma. Any research conducted in a multicultural society, such as Singapore, needs to be aware of how these shape public responses to science. For example, Yang et al identified three stages that a stigma goes through as it evolves: namely, "direct individual discrimination, internalisation of negative stereotypes, and structural discrimination". ${ }^{32}$ Stigma can also lead to social rejection, loss of employment prospects and exclusion from health insurance. ${ }^{33}$ As a result, Chinese Singaporeans are far more likely to hide their illness and not seek treatment compared to people of other major races in Singapore. ${ }^{34}$ Another long-lasting aspect of such stigma in Singapore is in the context of its consumer-orientated, medical savings account-based health system. This affects all kinds of decision-making in health care, and could continue to perpetuate stigmas about certain illnesses such as mental health conditions.

People who are 'unwell', or are identified as being at risk of developing such a disorder in the future, might face difficulties in academic and professional growth during their lifetime and exclusion from the rest of their community. These issues are important because, once contextualised alongside the ethical analysis of perceptions about genomic research in mental health, they can be addressed through careful micro- (between the researcher and participants) and macro-management, including public education and interaction with other parties. This has implications that all researchers should be aware of, including those involved with LYRIKS. However, these implications are also balanced out through positive effects of a diagnosis.

For example, our sample reveals that individuals with UHR are help-seeking - this is also highlighted in the fact that recruitment took place at the clinic for the IMH. This could be due to co-morbidities or a drop in functioning, thus they are already exposed to the stigma that goes with mental illnesses. An issue, highlighted recently with autism, is that in many societies, without a diagnosis treatment becomes impossible due both to ethical concerns as well as to insurance and to managed care issues. Thus for some, a diagnosis could be helpful.

In the following sections, we spell out the repercussions of genomic research in three phases, and expand upon the notion of risk by exploring perceptions held by the individual, the public, and the scientist. 


\section{The Individual's Perception of Risk}

While it is hoped that genomic research will provide genotypic indications that will allow for early identification and intervention in the prognosis of psychosis, such information is also combined with the individual's comprehension of the illness and their at-risk status. An individual is a relational being. As noted by Kleinman, a given medical system contains particular conceptualisations about the human body and does "considerably more than name, classify, and respond to illness" when it is placed in its socio-cultural context - an inevitable feature of the body as instrumental of the 'person'. ${ }^{35}$ Thus, the implications of statistical probability and clinical diagnosis affect not only the belief systems surrounding the nature of cognition and conceptualisation, but also relationships and roles that influence the perception of 'risk'. The perception of risk for the individual is then shaped by certain consequences of them being clinically classified as high-risk, which must make inferences about the individual's specific context of family and society - the kind of support they receive, whether home life is supportive or intolerable, their day-to-day stress, and exposure to social and environmental triggers. Therefore, there will likely be divergence between a clinical UHR state and an individual's perception of their own well-being that psychiatric management must grasp for optimal treatment.

It follows that the manageable risks that an individual faces personally may become a component of a wider 'at-risk' population who may now be seen as 'abnormal' and thus a 'threat' to society. Overall, the long-term aim is to counteract negative perceptions of mental illness in society by finding a shared understanding of mental health and developing appropriate measures to manage illness within a community that involves the individuals themselves, professionals (scientists and clinicians), and community representatives, and by educating the public that 'abnormal' mental health is not inevitably to be feared. An initial negative perception of risk can change for the individual following the emergence of a more aware, understanding and sympathetic 'public'.

The relationship between research and clinical settings also requires control over potential ethical conflicts - a concern that is typified in, for example, the "therapeutic misconception'. McGorry et al argue that the ethical dilemmas in early intervention in psychiatry are similar to those occurring in mainstream medicine. ${ }^{36}$ The prejudice that an individual may face, both from medical professionals and the public, is a result of segregating psychiatry and psychiatric research as qualitatively different from other medical research, ${ }^{37}$ even though the Diagnostic and Statistical Manual of Mental Disorders has (not uncontroversially) established the biomedical model of mental illness. The division has been widely criticised, for example, by Ivan Illich in the 1970s, who argued that the medical establishment of the West - a medical system that prioritises an increasingly segregated and biological (natural) view of medicine poses a threat to health by causing individuals to divorce from sickness, pain, and death. $^{38}$

While the increasing medicalisation of disease warrants its own critique, the relevant 
point for our discussion is that it can also act as a neutraliser in judgements and preconceptions about what disease and disease risk means. Because this naturalism has yet to infiltrate fully into the domain of psychiatry, psychiatric research and clinical psychiatry are not immune to stigmatisations that are prevalent in certain societies and this has particular relevance to understandings of risk. However, it is only through continued analysis into understanding the risks involved in genomic research about psychosis that knowledge will be gained about clinical management, which in turn may improve perceptions about psychiatric disorders.

Therefore, the design of the LYRIKS requires us to consider the potential use of genomic data on psychosis in the clinical setting with respect to the individual's comprehension of 'at-risk', and potential ethical conflicts. Importantly, as recognised in the design of the study, some of the participants may require clinical intervention during the study because of emerging symptoms and co-morbidity. These possibilities illustrate the 'risks' that are revealed during the prodromal state and the subsequent researcher-clinician responsibilities to act upon the clinical needs of the patients. This issue is acknowledged in the research protocol. ${ }^{39}$

\section{Public Perceptions of Genomic Data.}

Public perceptions of genomic data, based on society's collective understanding, are an integral part of an individual's decision-making in terms of determining risk (whether to undergo a test) and diagnosis (the conduct of society in respect to an atrisk condition). Concerns arise over the social consequences that result from how information is presented, especially in the context of the implications of identifying the genetic components of, for example, psychosis, which relate the mental state of the individual to his or her biology and history of epigenetic events. It is important to achieve a fine balance between over-simplifying genomic analysis and allowing such data to be accessible in a way that enables it to have a positive function for the public.

Yet, increased scientific and clinical knowledge of risks associated with psychosis acts as an important caveat to therapeutic translation. In other words, lifestyle is contextualised within the realm of a standardised and objectified body ${ }^{40}$ leading to a stronger relationship between individual experiences and the clinical encounter. This raises important implications for psychiatric treatment. Improving health outcomes by utilising genomic data is a significant function of healthcare. While individuals who are identified as being UHR may not necessarily develop a psychotic disorder, identifying features of this phase in terms of behaviour and corroborating it with genomic data may provide clinicians with better predictive tools that may have diagnostic utility. ${ }^{41}$ Consequently, such research improves clinical practice, which increases public confidence and improves the environment in which the UHR individual is supported.

This current clinical ambivalence about UHR diagnoses also impacts the translation of genomic data into the public arena and influences public opinion. Identifying a genetic causal basis of psychiatric disorders isolates genomic data as the source of an 
individual's classification as mentally 'healthy' or 'ill'. Consequently, the labels that arise in public perceptions of psychiatric disorders are potentially tied to the genomic data that defines the boundaries between 'normality' and pathology. The public perception of genomic data can have a positive effect, in the sense that there is a greater awareness and understanding of mental illness and its various causal factors. In turn, these factors play a role in encouraging and supporting individuals to seek early clinical intervention and thereby prevent further deterioration.

However, there are also negative perceptions when genomic data is associated with the presence of certain mental illnesses. For example, 88 per cent of participants in a USA nationwide survey interpreted genomic data to be an actual diagnosis rather than an assessment of risk for potentially developing schizophrenia. ${ }^{42}$ This can lead to immediate misconceptions, which are often stereotypical and related to prejudice against individuals with mental illness. Responsible communication about the nature of genomic data is crucial.

\section{Scientists' Perceptions of Genomic Data and the Clinical Setting.}

Finally, we turn our attention to the researcher creating the data. Some of the initial perceptions and responsibilities associated with genomic data stem from the laboratory. With the advent of validated genomic data, the involvement of genetic markers is adding to the multifactorial definition of psychosis. In turn, there is a greater emphasis on the relationship between genomic data and scientists' perceptions. This is an important point to consider, because within the scientific community there are plural and diverse opinions regarding the use of genomic data, and the challenge of developing a unified viewpoint may have further consequences. For example, the extent to which genomic data is considered as a fundamental indicator of risk for psychosis will affect diagnostic criteria. More specifically, the presence of certain gene markers will contribute to defining an individual as being 'mentally ill'.

Furthermore, a scientific understanding of the nature of genomic data affects the classification of the illness as 'chronic' or 'recoverable', as well as available treatment interventions. This is a difference between determinism and treatment. Importantly, identifying UHR individuals may overcome stigma because treatment and management are opportunities. Moreover, one could avoid the kind of malaise that suppresses a desire for treatment, allowing the condition to deteriorate unnecessarily.

The solution is to get patients involved with their treatment by employing an 'operational definition, ${ }^{43}$ that takes into account personal factors, such as the person's relationships and networks, as vital components in remission and/or recovery. A recent development is for researchers to involve participants in research, thus encouraging commitment to a long-term project and raising the participant's awareness. ${ }^{44}$ This was a major consideration in the LYRIKS study and efforts were made in the protocol to make sure that participation was possible, including the involvement of other family members (particularly parents and guardians who would 
be required to proxy-consent for participation by minors) where appropriate and desirable.

Predictive medicine, formed on the basis of scientist's interpretation of genomic data, is also associated with a responsibility to prevent perceptions of 'bad' genes or reductionist thinking from taking hold. This is particularly crucial when these perceptions leave the laboratory, such as in the context of third party handlers of an individual's genetic data, including health insurance companies or employers. In the prevention of risk, any potential negative implications of identifying genetic markers for psychiatric disorders are initially the responsibility of the scientist.

However, other clinical team members also play a part in the management and communication of genomic information. There is a public interest in genomic screening if it has a sound scientific basis and a clear rationale. For example, the American Medical Association recommends that testing for an untreatable genetic illness in children should wait until they are old enough to consent (American Medical Association; Opinion 2.131, 2.137, and 2.138). However, this view is contentious. One study researching attitudes towards a prospective genetic test for susceptibility to major depression showed that for the number of those resisting the test decreased by over a third following a facilitated discussion about positive and negative outcomes. Significantly, the participants who were prepared to undergo such a test stressed that an implicit trust in medical professionals would be required. ${ }^{45}$ This further emphasises that appropriate communication by members of clinical teams and the translations of the scientist's perceptions are necessary for counteracting the risks drawn out in our paper about genomic research on psychosis.

\section{Conclusion}

In this paper, we have explored the understanding of risk associated with genomic research and the possibility of identifying individuals who are at UHR of developing psychosis. We contextualised such research by referring to a current study in Singapore and developed a perspective that the nature of risk involved with genomic research and testing may be constructed from social and cultural processes.

Psychiatric disorders such as schizophrenia are associated with particular stigmas, which influence an individual's decision-making. We analysed the perceptions of the individual, the public, and scientists about risks associated with a UHR diagnosis.

Questions about genetic determinism and how this is irrevocably linked to stigmatisation and discrimination are familiar. Such perceptions underpin the ethical issues that accompany proposals such as the identification of UHR individuals. However, this paper, as a backdrop for further discussion, raises the importance of ensuring that scientific understandings of mental illness are not constructed in such a way that the dogmatisms of genetic determinism are revisited. This not only requires a sophisticated approach to communicating to the public the predictive limits of genomic research in terms of risk profiling, but also requires scientists to be more acutely aware of the values that are attached to scientific knowledge, as well as the 
authority it carries in the public domain, which is inherently political and value-laden. Genomic research, then, should focus upon relational aspects in society, and the objectification of risk amongst individuals.

${ }^{1}$ University College London Medical School; Centre for Biomedical Ethics, National University of Singapore; Human Genetics Genome Institute of Singapore; Institute of Mental Health, Singapore; National Medical Research Council, Singapore; Centre for Biomedical Ethics, National University of Singapore. Correspondence to: a.ahmad@ucl.ac.uk

${ }^{2}$ Conus P, Ward J, Hallam K.T, Lucas N, Macneil C, McGorry P.D, Berk M. 2008. 'The Proximal Prodrome to First Episode Mania - A New Target For Early Intervention'. Bipolar Disorders. 10 (5): 555-565.

${ }^{3}$ Yung A.R, Nelson B, Thompson A. 2010. 'The Psychosis Threshold in Ultra High Risk (prodromal) Research: Is it valid?' Schizophrenia Research. 120: 1-6.

${ }^{4}$ Ruhrmann S, Schultze-Lutter F, and Klosterkotter J. 2010. 'Probably At-Risk, But Certainly Ill Advocating the Introduction of a Psychosis Spectrum Disorder in DSM-V'. Schizophrenia Research 120 (1-3): 23-37.

${ }^{5}$ Phillips L.J., McGorry P.D., Yung, A.R. 2005. 'Prepsychotic phase of schizophrenia and related disorders: Recent progress and future opportunities'. British Journal of Psychiatry: 187.

${ }^{6}$ Addington J, Leriger E, Addington D. 2003. 'Symptom outcome 1 year after admission to an early psychosis programme'. Canadian Journal of Psychiatry; 48: 204-207.

${ }^{7}$ Petrie K.J., Weinmann J. 2006. 'Why illness perceptions matter'. Clinical Medicine; 6: 536-539.

${ }^{8}$ McGlashan T.H, Addington J, Cannon T, Heinimaa M, McGorry P, O’Brien M, Penn D, Perkins D, Salokangos R.K.R, Walsh B, Woods S.W, Yung A. 2007. 'Recruitment and Treatment Practices for Help-Seeking "Prodromal Patients"'. Schizophrenia Bulletin; 33 (3): 715-726.

${ }^{9}$ WHA 67.13: 2004. 'Genomics and World Health'. Fifty Seventh World Health Assembly Resolution.

${ }^{10}$ Sotiriou C, and Piccart M.J. 2007. 'Taking Gene-Expression Profiling to the Clinic: When Will Molecular Signatures become Relevant to Patient Care?' Nature Reviews Cancer 7: 545-553.

${ }^{11}$ Hall, W., and Pacula R.L. 2003. Cannabis Use and Dependence: Public Health and Public Policy. Cambridge University Press.

${ }^{12}$ Frieden, J. 'Use of Racially Targeted Drug Therapy Questioned'. 2006. Published online: www.clinicalpsychiatrynews.com.

${ }^{13}$ Veatch, R. 1989. Cross cultural perspectives in medical ethics: Readings. Boston, MA: Jones and Bartlett.

${ }^{14}$ Link BG. 1982. 'Mental patient status, work, and income: An examination of the effects of a psychiatric label'. American Sociological Review; 47: 202-215.

${ }_{15}$ Jorm A.F, Korten A.E, Jacomb P.A, Christensen H, Rodgers B, Pollitt P. 1997. 'Public Beliefs about Causes and Risk Factors for Depression and Schizophrenia'. Social Psychiatry and Psychiatric Epidemiology: 32(3):143-8.

${ }^{16}$ Economou M.P, Stefanis N.C, and Papadimitriou G.N. 2009. 'Schizophrenia and Stigma: Old Problems, New Challenges'. In Schizophrenia. S. Kaspar and G.N. Papadimitriou, eds. London: Informa: 299-309.

${ }^{17}$ Walker I, and Read J. 2002. 'The Differential Effectiveness of Psychosocial and Biogenetic Causal Explanations in Reducing Negative Attitudes toward "Mental Illness"'. Psychiatry: Interpersonal and Biological Processes 65(4): 313-325.

${ }^{18}$ Smerecnik C., Grispen J.E.J., Quaak M. 2011. 'Effectiveness of Testing for Genetic Susceptibility to Smoking-Related Diseases on Smoking Cessation Outcomes: A Systematic Review and MetaAnalysis'. Tobacco Control 2012, 21(3): 347-354.

${ }^{19}$ Hall WD (2005) Will Nicotine Genetics and a Nicotine Vaccine Prevent Cigarette Smoking and Smoking-Related Diseases? PLoS Med 2(9).

${ }^{20}$ Yung A.R, Nelson B, Thompson A. 2010. 'The Psychosis Threshold in Ultra High Risk (prodromal) Research: Is it valid?' Schizophrenia Research. 120: 1-6.

${ }^{21}$ Craddock N, O’Donovan M.C., and Owen M.J. 2007. 'Phenotypic and Genetic Complexity of Psychosis: Invited Commentary on Schizophrenia: A Common Disease Caused by Multiple Rare Alleles'. The British Journal of Psychiatry: 190: 200-203. 
${ }^{22}$ McGlashan T.H, Addington J, Cannon T, Heinimaa M, McGorry P, O’Brien M, Penn D, Perkins D, Salokangos R.K.R, Walsh B, Woods S.W, Yung A. 2007. 'Recruitment and Treatment Practices for Help-Seeking "Prodromal Patients". Schizophrenia Bulletin; 33 (3), p. 715-726.

${ }^{23}$ Lysaght T, Capps, B.J, Campbell, A.V, Subramaniam M. \& Chong, S. 2011. 'Intervening in Clinical Research to Prevent the Onset of Psychoses: Conflicts and Obligations'. Journal of Medical Ethics, Advanced online publication: doi:10.1136/medethics-2011-100008.

${ }^{24}$ Corcoran C, Malaspina D, Hercher L. 2005. 'Prodromal Interventions for Schizophrenia Vulnerability: The Risks of Being At Risk'. Schizophrenia Research 73(2): 173-184.

${ }^{25}$ Kevles, D. 1985. In the name of eugenics: Genetics and the uses of human heredity. Los Angeles: University of California Press.

${ }^{26}$ Pinker, S. 2002. The blank slate: The modern denial of human nature. London: Penguin.

${ }^{27}$ Meaney M. 2001. 'Nature, Nurture, and the Disunity of Knowledge'. Annals New York Academy of Sciences: 935:50-61.

${ }^{28}$ Holm, S., and Rossel, P. 1999. 'How does the public perceive the motives of medical researchers for doing research?' Bulletin of Medical Ethics 146: 16-17.

${ }^{29} \mathrm{Ng}$, P.C, Zhao Q, Levy S, Strausberg R.L, and Ventre J.C. 2008. 'Individual Genomes Instead of Race for Personalized Medicine'. Clinical Pharmacology and Therapeutics 84: 306-309.

${ }^{30}$ Tutton, R, Smart A, Martin P.A, Ashcroft R, Ellison G.T.H. 2001. 'Genotyping the Future:

Scientists' Expectations about Race/ Ethnicity after BiDil'. American Society of Law, Medicine, Ethics 36 (3): 464-470.

${ }^{31}$ Link, B.G., Yang, L.H., Phelan, J.C., and Collins, P.Y. 2004. 'Measuring mental illness stigma'. Schizophrenia Bulletin 30( 3).

${ }^{32}$ Yang, L.H., Wonpat-Borja, A.J., Opler, M.G., Corcoran, C.M. 2010. 'Potential stigma associated with inclusion of the psychosis risk syndrome in the DSM-V: An empirical question'. Schizophrenia Research; 120 (1-3): 42-48.

${ }^{33}$ Lai YM, Hong CPH, Chee CYI. 2001. 'Stigma of Mental Illness'. Singapore Medical Journal: 42(3):111-4.

${ }^{34}$ Chong S, Verma S, Vaingankar J, Chan Y, Wong L, Heng B. 2007. 'Perception of the Public Towards the Mentally Ill in a Developed Asian Country'. Social Psychiatry and Psychiatric Epidemiology. [10.1007/s00127-007-0213-0]. 42(9):734-9.

${ }^{35}$ Good, B.J., Fischer, M.M.J., Willen, S.S., and Good, M.J.D. 2010. A reader in medical anthropology: Theoretical trajectories, emergent realities. Oxford: John Wiley and Sons.

${ }^{36}$ McGorry, P.D., Killackey, E., and Yung, A. 2008. 'Early intervention in psychosis: Concepts, evidence, and future directions'. World Psychiatry; 7(3): 148-156.

${ }^{37}$ Hodge, Jr J. 2004. 'Ethical Issues Concerning Genetic Testing and Screening in Public Health'. American Journal of Medical Genetics 125C (1): 66-70.

${ }^{38}$ Illich, I. 1976. Limits to Medicine: Medical Nemesis: The Expropriation of Health. London: Pantheon Books.

${ }^{39}$ Lysaght T, Capps, B.J, Campbell, A.V, Subramaniam M. \& Chong, S. 2011. 'Intervening in Clinical Research to Prevent the Onset of Psychoses: Conflicts and Obligations'. Journal of Medical Ethics. Advanced online publication: doi:10.1136/medethics-2011-100008.

${ }^{40}$ Lock, M., and Nguyen, V.K. 2010. An anthropology of biomedicine. Oxford: John Wiley and Sons.

${ }^{41}$ Tsuang MT, Nossova N, Yager T, Tsuang MM, Guo SC, Shyu KG, et al. 2005. 'Assessing the validity of blood-based gene expression profiles for the classification of schizophrenia and bipolar disorder: a preliminary report'. American Journal of Medical Genetics Neuropsychiatry Genetics 133:1-5.

${ }^{42}$ Link, BG., and Phelan, J.C. 2001. 'Conceptualising Stigma'. Annual Review Sociology; 27: 363-85.

${ }^{43}$ Liberman, R.P., Kopelowicz, A., Ventura, J., and Gutkind, D. 2002. 'Operational criteria and factors related to recovery from schizophrenia'. International Review of Psychiatry; 14: 256-272.

${ }^{44}$ Campbell A. 'The Ethical Challenges of Genetic Databases: Safeguarding Alturism and Trust'. 2007. King's Law Journal, 18 (2): 227-245.

${ }^{45}$ Wilde, A., Meiser, B., Mitchell, P.B., and Schofield, P.R. 2010. 'Public interest in predictive genetic testing, including direct-to-consumer testing, for susceptibility to major depression: preliminary findings'. European Journal of Human Genetics; 18: 47-51. 